\title{
CLINICAL EFFECTIVENESS OF TREATMENT THE PATIENTS WITH CHRONIC APICAL PERIODONTITIS
}

\author{
N. G. Gadzhula \\ NATIONAL PIROGOV MEMORIAL MEDICAL UNIVERSITY, VINNYTSIA, UKRAINE
}

\begin{abstract}
Background. The success of endodontic treatment is provided by a thorough instrumental and antiseptic treatment of infected root canals, and it depends on the composition of filling material, the degree of adhesion to dentin, hermetic obturation of apical foramen, solubility of sealer.

Objective. The study was aimed to evaluate the effectiveness of root canal obturation with BioRoot ${ }^{T M} R C S$ sealer in the treatment of patients with chronic apical periodontitis.

Methods. Endodontic treatment of 23 teeth in 20 patients with chronic apical periodontitis by method of lateral compaction of gutta-percha was carried out. In the main group root canals were obturated with BioRoot ${ }^{T M}$ $R C S$, in the control group the canals were filled with Apexit Plus. The percentage of efficient or non-efficient cases was evaluated on the basis of radiographic comparison of treated chronic apical periodontitis immediately after obturation, in three, six months and one year. Radiographic conditions were defined as existing state, improvement and worsening.

Results. In a year of dynamic evaluation the final results were: in the main group - 54.55\% of the patients had complete bone healing, in $27.27 \%$ of cases the focus of bone destruction was decreased by $1 / 2$ or more of the initial sizes, $18.18 \%$ - resorption lesion was decreased by less than 1/2; in the control group - 33.33\% of improvement, $25.0 \%$ of existing state and $41.66 \%$ of worsening.

Conclusions. BioRoot ${ }^{T M}$ RCS using for root canals obturation in the treatment of chronic apical periodontitis we proved the high effectiveness of the treatment undertaken: complete healing or improvement of radiographic conditions of periapical bone destruction with X-ray signs of bone regeneration.
\end{abstract}

KEY WORDS: apical periodontitis, endodontics, sealers, filling, root canals.

\section{Introduction}

Treatment of patients with chronic apical periodontitis is complex and is an important task of modern dentistry due to their high prevalence, frequent complications after traditional treatment and absence of consistent results [1,2]. According to numerous researches, the basic principles of effective treatment of destructive forms of apical periodontitis are the thorough instrumental and antiseptic treatment of infected root canals with their subsequent filling with pastes based on calcium hydroxide $[3,4]$. It prevents microbial contamination and its influence on surrounding tissues, acceleration the processes of apexification and bone regeneration $[5,6]$. The success of endodontic treatment is also impossible without quality

Corresponding author: Nataliia Gadzhula

Therapeutic Dentistry Department, National Pirogov Memorial Medical University, Pirogov Street 52, Vinnytsia, 21001 Phone number: (0432) 61-85-79; 097-701-19-18

E-mail: natalidentist1@gmail.com hermetic obturation of root-canal system for forming of reliable barrier between tooth cavity and periodontal ligament $[1,2,6]$.

Among all modern endodontic sealers a mineral-based root canal sealer BioRoot ${ }^{\mathrm{TM}} \mathrm{RCS}$ "Septodont" has the above-mentioned features. This material is bioactive by stimulating bone physiological process and mineralization of dentinal structure [7]. It creates a favourable environment for periapical healing and bioactive features including biocompatibility, hydroxyapatite formation, mineralization of dentinal structure, alkaline $\mathrm{pH}$ and sealing qualities. Due to the use of Active BioSilicate Technology which is monomer free, there is no shrinkage of BioRoot ${ }^{\mathrm{TM}} \mathrm{RCS}$ during setting for a tight seal of root canal $[8,9]$.

Therefore, this study was aimed to evaluate the effectiveness of root canal obturation with BioRoot ${ }^{\mathrm{TM}}$ RCS sealer in the treatment of patients with chronic apical periodontitis. 


\section{Material and Methods}

Endodontic treatment of 23 teeth from frontal and distal groups with chronic apical periodontitis (by an equal distribution between chronic granulating and granulomatous periodontitis) in 20 patients aged 25-45 years without accompanying pathology was carried out. Disinfection and irrigation procedures of root canals were made by standard methods: the canals were shaped and underwent an appropriate tapered preparation, disinfected with a $3 \%$ sodium hypochlorite solution activated with mechanical agitation and rinsed with $17 \%$ EDTA. Permanent obturation of root canals was carried out by method of lateral compaction of gutta-percha. In the main group the root canals of 11 teeth were obturated with BioRoot ${ }^{\mathrm{TM}}$ RCS "Septodont" as a sealer, in the control group the root canals of 12 teeth were filled with Apexit Plus "Ivoclar Vivadent".

The percentage of efficient or non-efficient cases was evaluated by radiographic comparison of treated chronic apical periodontitis immediately after obturation, and in three, six and twelve months. Radiographic conditions were defined as existing state, improvement and worsening. Dynamics of periapical tissue healing were also controlled by a periapical index PAI [10].

Descriptive statistics was compiled by Microsoft Excel and Student's t-test. Mann-Whitney $U$-test (nonparametric criteria) was used to evaluate differences between groups at baseline (immediate postoperative) and at the 12-month follow-up evaluation. Changes in PAI score for each group from baseline to 12-month follow-up evaluation were tested with Wilcoxon signed rank test. The secondary outcome measures, proportion of teeth in each group that could be considered as improved (PAI score decreased) or healed (PAI $<2$ ) were assessed with Chi-square test [3]. Clinical symptoms and abnormal findings at the follow-up examination were recorded but not subjected to statistical analysis.

\section{Results}

By using BioRoot ${ }^{\mathrm{TM}} \mathrm{RCS}$ for nonsurgical treatment of destructive forms of chronic apical periodontitis in the patients of the main group we did not observe any complications after contact with this material. In three months after the treatment was completed the dynamics of clinical and radiographic changes were practically identical in both groups of patients, although complete healing of periapical bone area was not evidenced in any clinical cases. The average values of PAI before and after 3 months of the treatment did not differ significantly in the study groups of the patients $(p>0.05)$.

In 6 months the patients of the main group were absolutely asymptomatic. Clinical symptoms or abnormal findings (spontaneous pain, swelling, mobility, or sensitivity to percussion or palpation) in the main group of patients were absent. In 2 patients of the control group the exacerbation of chronic apical periodontitis was diagnosed and the teeth were re-treated. Intergroup significant differences of PAI were determined after 6 months of dynamic observation: in the main group $-2.09 \pm 0.26$ points, in the control group $-2.92 \pm 0.31$ points $(p<0.05)$.

In six months on the X-ray images of treated teeth in the main group of patients the absence of periapical zone destruction was determined in $36.36 \%$ of cases (4 teeth) and reduction of focus of apical bone resorption by $1 / 2$ or more was established in $45.45 \%$ (5), the existing state was in $18.18 \%$ (2). In the control group in six months after root canal obturation the widening of periapical destruction zone was revealed in $25.0 \%$ (3) of cases, the focus of bone destruction was not changed in $33.33 \%$ (4), it was decreased by less than $1 / 2$ in $25.0 \%$ (3), and it was decreased by $1 / 2$ or more from the initial size in $16.67 \%(2)$.

12-month follow-up examinations proved that the patients of the main group did not have any complaints. During intraoral examination the gingiva in the area of tooth apexes was of normal colour without visible pathological changes, except scar formation from gingival fistula after the treatment of chronic granulating periodontitis. Percussion reaction was painless, regional lymph nodes were not palpable. The teeth performed full functional load. Mean PAI score in the main group was $3.27 \pm 0.43$ at baseline and decreased up to $1.55 \pm 0.35$ in 1 year of observation, in the control group it was $3.25 \pm 0.39$ and $2.75 \pm 0.38$, respectively. The decrease in PAI in the main group was up to 1.72, in the control group - up to 0.5. The decrease in PAI was statistically significant in both groups of the patients $(p<0.001)$.

In a year of dynamic assessment of the main group the improvement of radiographic state was evidenced: $54.55 \%$ (6) of the patients had complete bone healing, $27.27 \%$ (3) of cases the focus of bone destruction was decreased by $1 / 2$ or more of the initial sizes, $18.18 \%(2)$ - the resorption lesion was decreased by less than 
$1 / 2$. Final results in the control group of patients were: $33.33 \%$ (4) of improvement, $25.0 \%$ (3) of existing state and $41.66 \%$ (5) of worsening. The absence of positive dynamics during 12 months of observation of the main group patients was in $18.18 \%$ (2) of cases; the percentage of non-efficient cases in the control group was $41.66 \%$ (5).

\section{Discussion}

Repair of periradicular tissues consisted of a complex regeneration involving bone, periodontal ligament, and cementum. Although clinical as well as radiographic data were used to monitor cases, the relative absence of clinical symptoms in chronic apical periodontitis made the evaluation primarily a radiographic one [10]. Due to the use of BioRootTM RCS in the main group of patients the positive dynamics of clinical manifestations was confirmed radiographically during periodic follow-up examinations. Three months after the treatment was completed we revealed the lesions size reduction, increasing of the number of bone trabecules and decreasing of intertrabecular spaces. In six months of BioRootTM RCS exposition in root canals the expansion of area destruction in periapical tissues and the appearance of new rarefaction or the increase in initial rarefaction were not determined in any clinical case. On X-ray images bone thickening was observed, periodontal contours were not widened. Moreover, in six months the radiographic evidence of trabecular pattern of bone was determined at the area the periradicular lesion was present before. In a year of dynamic assessment a significant thickening of bone and regeneration of periapical tissues were evidenced. The contour, width, and structure of periodontal margin were normal. The area of mineral loss gradually was filled with bone and the radiographic density was increased in most clinical cases.

\section{References}

1. Siqueira JF, Rocas IN, Ricucci D, Hulsmann M. Causes and management of post-treatment apical periodontitis. British Dental J 2014; 216: 305-312.

2. Yu VS, Khin LW, Hsu CS, Yee R, Messer HH. Risk Score Algoritm for Treatment of persistent apical periodontitis. J Dent Res 2014; 93 (11): 1076-1082.

3. Gill GS, Bhuyan AC, Kalita C, Das L, Kataki R, Bhuyan D. Single versus multi-visit endodontic treatment of teeth with apical periodontitis: An in vivo
Composition of BioRootTM RCS (bioactive tricalcium silicate setting system with the absence of any aluminate and calcium sulphate), high degree of adhesion to the dentin and gutta-percha points, zero shrinkage as monomer-free sealer and great flowability created a favourable environment for periapical healing, the acceleration of apexification and regeneration processes of the bone, and provided a hermetic obturation of apical foramen [9]. There were no complications after the conducted treatment.

Comparing the clinical and radiographic results of both groups it should be noted that in the patients of the main group BioRoot ${ }^{\mathrm{TM}} \mathrm{RCS}$ usage resulted in the improvement of clinical symptoms of the disease. Repair of periapical bone structures and periodontal tissues, increasing of mineral saturation of bone were accelerated by this sealer, especially it was a characteristic feature of pathological processes with size of destruction up to $3 \mathrm{~mm}$ in diameter. Regeneration in these clinical cases occurred faster than in the cases of larger sizes of destructive lesions. Although the action of BioRoot ${ }^{\mathrm{TM}} \mathrm{RCS}$ was also effective in the patients with the large periapical lesions, but in the control group the healing of such lesions was not evidenced.

\section{Conclusions}

BioRoot ${ }^{\mathrm{TM}}$ RCS using for root canal obturation in the treatment of chronic apical periodontitis we proved the effectiveness of the treatment undertaken: complete healing or improvement of radiographic states of periapical bone destruction with X-ray signs of bone regeneration. These changes were confirmed by the higher indicators of radiographic dynamics of periapical index in the main group of patients than in the other. study with 1-year evalution. Annals of Medical \& Health Sciences Res 2016; 6: 19-26.

4. Tyagi S, Mishra P, Tyagy P. Evolution of root canal sealers: An insight story. European J of Dentistry 2013; 2 (3): 199.

5. Murugesan Gawthaman, Selvarai Vinodh, Veerabadhran Mahesh Mathian, Rangasamy Vijayaraghavan, Ramachandran Karunakaran. Apexification with calcium hydroxide and mineral trioxide aggre- 
gate: report of two cases. J Pharm Bioallied Sci 2013: 5 (2): 131-134.

6. Nair PNR. On the causes of persistent apical periodontitis: a review. International Endod J 2006; 39: 249-281.

7. Camps J, Jeanneau C, EI Avachi I, Laurent P. Bioactivity of a calcium silicate-based endodontic cement (BioRoot RCS): interactions with human periodontal ligament cells in vitro. J Endod 2015; 41 (9): 1469-1473.
8. Zhang W, Li Z, Peng B. Ex vivo cytotoxicity of a new calcium silicate based canal filling material. International Endod J 2010; 42 (9): 769-774.

9. Simon S, Flouriot AC. BioRoot ${ }^{\mathrm{TM}}$ RCS a new biomaterial for root canal filling. J Case Studies Collection 2016; 13: 4-11.

10. Orstavik D, Kerekes K, Eriksen HM. The periapical index: a scoring system for radiographic assessment of apical periodontitis. Endod Dent Traumatol 1986; 2 (1): 20-34. 